\title{
A local density functional for the short-range part of the electron-electron interaction
}

\author{
Lorenzo Zecca, Paola Gori-Giorgi,* Saverio Moroni, and Giovanni B. Bachelet \\ INFM Center for Statistical Mechanics and Complexity, and Dipartimento di Fisica, \\ Università di Roma "La Sapienza," Piazzale A. Moro 2, 00185 Rome, Italy
}

(Dated: June 28, 2018)

\begin{abstract}
Motivated by recent suggestions -to split the electron-electron interaction into a short-range part, to be treated within the density functional theory, and a long-range part, to be handled by other techniques- we compute, with a diffusion Monte Carlo method, the ground-state energy of a uniform electron gas with a modified, short-range-only electron-electron interaction $\operatorname{erfc}(\mu r) / r$, for different values of the cutoff parameter $\mu$ and of the electron density. After deriving some exact limits, we propose an analytic representation of the correlation energy which accurately fits our Monte Carlo data and also includes, by construction, these exact limits, thus providing a reliable "short-range local-density functional".
\end{abstract}

\section{INTRODUCTION AND SUMMARY OF RESULTS}

Density functional theory ${ }^{1.2 .3}$ (DFT) is nowadays the most widely used method for electronic structure calculations, in both condensed matter physics and quantum chemistry, thanks to the combination of low computational cost and remarkable accuracy for a wide variety of chemical bonds and solid-state systems. There are, however, notable exceptions to such an accuracy. For example, even the best available approximations of the exchange-correlation functional, the key ingredient of the DFT, fail to recover long-range van der Waals interactions,, .5 .6 are not completely safe for the description of the hydrogen bond ${ }^{7}$ and have intrinsic problems with situations of near degeneracy (when two sets of orbitals happen to have very close energies) $\stackrel{8.9}{*}$ More generally, the "chemical accuracy" (the accuracy needed to predict the rates of chemical reactions) has not yet been reached. For all these reasons the search for new approximate functionals, or even new ways of exploiting the basic ideas and advantages of the DFT, is very active. 2,4,5.6.7.9

In this context several authors $8.9,10,11$ have suggested to split the electron-electron interaction into a shortrange part, to be treated within the DFT, and a longrange part, to be handled by other techniques. The motivation behind these "mixed schemes" is that the DFT, even in the simplest local-density approximation (LDA), provides an accurate description of the shortrange electron-electron repulsion, ${ }^{12}$ while other techniques which give a poor description of short-range properties, like the configuration interaction (CI) method or the random-phase approximation (RPA), 13.14 can, instead, accurately capture long-range correlation effects.

Of course there is no unique way to split the Coulomb potential $1 / r$ into a short-range (SR) and a long-range

\footnotetext{
* present address: Laboratoire de Chimie Théorique, Université Pierre et Marie Curie, Paris, France
}

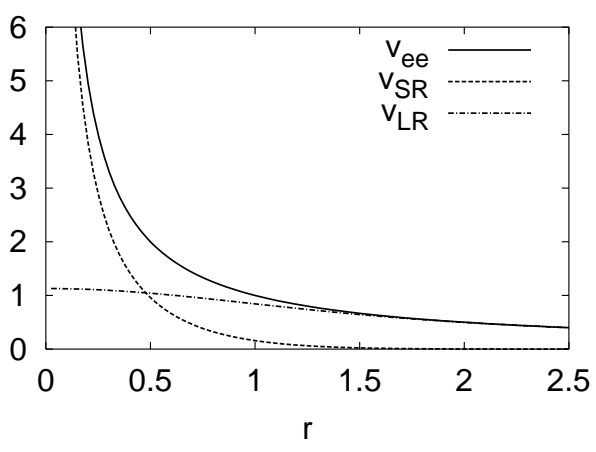

FIG. 1: Splitting of the Coulomb electron-electron repulsion $v_{e e}=1 / r$ into a short-range (SR) part and a long-range (LR) part, according to Eqs. (1)-(2), with $\mu=1$.

(LR) part. The error function and its complement

$$
\begin{aligned}
& v_{e e}(r)=\frac{1}{r}=v_{\mathrm{SR}}(r)+v_{\mathrm{LR}}(r), \\
& v_{\mathrm{SR}}(r)=\frac{\operatorname{erfc}(\mu r)}{r}, \\
& v_{\mathrm{LR}}(r)=\frac{\operatorname{erf}(\mu r)}{r},
\end{aligned}
$$

have been already used for this purpose ${ }^{8,9,11}$ (see Fig. 1), and we stick to this choice, which yields analytic matrix elements for both gaussians and plane waves, i.e., the most common basis functions in quantum chemistry and solid-state physics, respectively. This form still leaves room for some arbitrariness: the choice of the most convenient cutoff parameter $\mu$, which may be different for different "mixed schemes".

The combination of a short-range DFT calculation and a different treatment of the long-range part of the electron-electron interaction can be founded on a rigorous basis through the adiabatic connection formalism 8.9 .15 ? Depending on the specific problem addressed (van der Waals forces, near-degeneracy,...), and thus on the particular approach to the long-range part of the electronelectron interaction, different "mixed schemes" have been proposed $8.9,10$ But in all of them, as in standard DFT, 
a crucial role is played by the exchange-correlation functional, which now must be built for a modified electronelectron interaction. The schemes of Refs. 1011 need a pure short-range functional, $E_{x c}^{\mathrm{SR}}[n]$, whose LDA version is given by

$$
E_{x c}^{\mathrm{SR}, \mathrm{LDA}}[n]=\int n(\mathbf{r}) \epsilon_{x c}(n(\mathbf{r}), \mu) d \mathbf{r},
$$

where $\epsilon_{x c}(n, \mu)$ is the exchange-correlation energy per electron of a uniform gas of density $n$ interacting with a short-range potential like Eq. (1). The value of $\mu$ in Eq. (3) can be either a constant, or, possibly, a convenient function of the density, $\mu=\mu(n(\mathbf{r})) \stackrel{16}{\underline{16}}$ The local functional $\epsilon_{x c}(n, \mu)$ is the quantity which we provide in this paper. We start from a jellium-like hamiltonian (in Hartree atomic units used throughout this work)

$$
H=-\frac{1}{2} \sum_{i=1}^{N} \nabla_{\mathbf{r}_{i}}^{2}+V_{e e}^{\mathrm{SR}}+V_{e b}^{\mathrm{SR}}+V_{b b}^{\mathrm{SR}},
$$

where $V_{e e}^{\mathrm{SR}}$ is the modified electron-electron interaction

$$
V_{e e}^{\mathrm{SR}}=\frac{1}{2} \sum_{i \neq j=1}^{N} \frac{\operatorname{erfc}\left(\mu\left|\mathbf{r}_{i}-\mathbf{r}_{j}\right|\right)}{\left|\mathbf{r}_{i}-\mathbf{r}_{j}\right|},
$$

$V_{e b}^{\mathrm{SR}}$ is, accordingly, the interaction between the electrons and a rigid, positive, uniform background of density $n=$ $\left(4 \pi r_{s}^{3} / 3\right)^{-1}$

$$
V_{e b}^{\mathrm{SR}}=-n \sum_{i=1}^{N} \int d \mathbf{x} \frac{\operatorname{erfc}\left(\mu\left|\mathbf{r}_{i}-\mathbf{x}\right|\right)}{\left|\mathbf{r}_{i}-\mathbf{x}\right|},
$$

and $V_{b b}^{\mathrm{SR}}$ is the corresponding background-background interaction

$$
V_{b b}^{\mathrm{SR}}=\frac{n^{2}}{2} \int d \mathbf{x} \int d \mathbf{x}^{\prime} \frac{\operatorname{erfc}\left(\mu\left|\mathbf{x}-\mathbf{x}^{\prime}\right|\right)}{\left|\mathbf{x}-\mathbf{x}^{\prime}\right|} .
$$

First we calculate the ground-state energy per electron of this model hamiltonian, as a function of the density parameter $r_{s}$ and of the parameter $\mu$, with a diffusion Monte Carlo method (Sec. III). Then we derive the asymptotic behaviors of the correlation energy $\epsilon_{c}\left(r_{s}, \mu\right)$ (Sec. IIII). On these grounds we finally (Sec. IV) present a convenient analytic parametrization of the correlation energy, thus following in the footsteps from quantum simulations of the regular jellium model to the best available LDA functionals $\stackrel{17,18,19,20}{0}$

\section{DMC CALCULATION OF THE GROUND-STATE ENERGY}

A local density functional for the short-range potential of Eqs. (5)-(7) should recover the Ceperley-Alder ${ }^{17}$ (CA) correlation energy for $\mu \rightarrow 0$. In this Section we outline the implications of this condition on the technical aspects of our calculation, which is in all respects a standard application of the diffusion Monte Carlo method in the Fixed Node approximation (FN-DMC) ${ }^{21}$

The FN-DMC method gives the energy $E_{F N}$ of the lowest-lying Fermionic eigenstate of the Hamiltonian which has the same nodes as the chosen trial function $\Psi_{T}$. The error in $E_{F N}$ is variational, and it vanishes as the nodal structure of $\Psi_{T}$ approaches the (unknown) nodal structure of the exact ground state. The simplest choice for the trial function of a homogeneous fluid ${ }^{17}$ is the Jastrow-Slater form, $\Psi_{T}(R)=J(R) D(R)$, where the symmetric Jastrow factor $J(R)=\exp \left[-\sum_{i<j} u\left(r_{i j}\right)\right]$ describes pair correlations, and $D$ is the product of one Slater determinant of plane waves $(\mathrm{PW})$ for each spin component $(R$ denotes the coordinates of all the particles). A better nodal structure is provided by the socalled backflow $(\mathrm{BF})$ wave function ${ }^{22}$

The method used in Ref. 17 is in principle exact: it starts from the FN solution and then it performs a "nodal relaxation", whereby the energy converges to the exact ground-state result. However, this second process is accompanied by an increasing statistical noise, which may hinder full convergence of the results. In practice, the results of Ref. 17 are between the FN energies recently calculated with $\mathrm{PW}$ and BF nodes ${ }^{22}$, and actually somewhat closer to the former. Since, on one hand, BF calculations are considerably more demanding, and, on the other, the most widely used local-density functionals are constructed to fit the quantum Monte Carlo results of Ref. 17, we choose to stick to the simple trial function with Slater determinants of plane waves. In this way our "short-range local-density functional" will continuously merge into the Ceperley-Alder ${ }^{17}$-based localdensity functionals as $\mu \rightarrow 0$.

All the other errors in the simulation can be controlled and eliminated. It is easy to ensure that the biases due to a finite time step and a finite population of walkers ${ }^{21}$ are much smaller than the statistical uncertainty of the CA results, which we set as our target precision. The number extrapolation is more delicate. We simulate $N$ particles in a cubic box with periodic boundary conditions, interacting via the potential of Eq. (1). Since for very small values of $\mu$ we rely on the analytic asymptotic behavior described in Sec. IIII the only simulations we need to do will deal with really short-range potentials, which we may safely treat using the minimum image convention ${ }^{23}$ The dependence of the energy on the number of particles is determined with the Variational Monte Carlo (VMC) method, which calculates the expectation value of the Hamiltonian operator on the trial wave function and is cheaper than DMC. For several values of $N$ (namely $38,54,66,114,162$ ), we use VMC to calculate (i) the variational energy $E_{V}$ (after optimization of the Jastrow factor), and (ii) the Hartree-Fock energy $E_{H F}$, which corresponds to $J=1$. For each value of $r_{s}$ and $\mu$, the resulting estimate of the correlation energy per electron, $\epsilon_{c}=\left(E_{V}-E_{H F}\right) / N$, is fitted to the following 


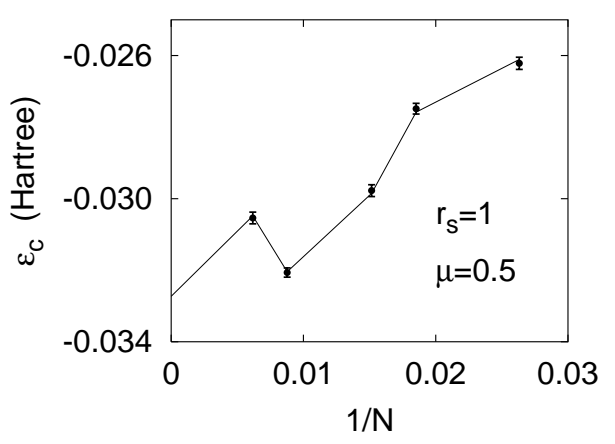

FIG. 2: Correlation energy per particle of the short-range interacting gas at $\mu=0.5 r_{s}=1$, for different numbers of particles $N$. The fitting function of Eq. (8) (line) favorably compares with the VMC data (dots).

form:

$$
\begin{aligned}
\epsilon_{c}\left(r_{s}, \mu ; N\right)= & \epsilon_{c}\left(r_{s}, \mu ; \infty\right)+a\left(r_{s}, \mu\right)[T(\infty)-T(N)] \\
& +b\left(r_{s}, \mu\right) / N
\end{aligned}
$$

Here $T(N)$ is the kinetic energy of $N$ non-interacting electrons at $r_{s}=1$, and $a\left(r_{s}, \mu\right), b\left(r_{s}, \mu\right)$ and the correlation energy in the thermodynamic limit, $\epsilon_{c}\left(r_{s}, \mu ; \infty\right)$ are fitting parameters. The size dependence of the VMC result for the correlation energy is shown in Fig. 2 for the case where it is largest (small $r_{s}$ and small $\mu$ ). We point out that the simple functional guess of Eq. (8) (solid line) accurately models the size dependence of the VMC data which, although on a small energy scale, are still far from a smooth dependence (dots with error bars). Our final result for the correlation energy is obtained by adding the infinite-size extrapolation obtained from Eq. (8) to the result of a single DMC simulation with $N=54$.

\section{ASYMPTOTIC BEHAVIORS}

In this section we derive some limiting behaviors of the correlation energy $\epsilon_{c}\left(r_{s}, \mu\right)$, which will be used for its parametrization in Sec. IV] The detailed study carried out here can be also of interest for the choice of a densitydependent $\mu$ parameter in the mixed schemes of Refs. $\underline{9}$ and 10 .

We consider two different regimes: when our system approaches the standard jellium model (i.e., full interaction $1 / r$ ), and when it approaches the noninteracting Fermi gas. In the first case (Secs. [IA and ЩB) we find that the correlation energy is a function of the scaled variable $\mu \sqrt{r_{s}}$, while in the second case (Sec. IIIC) the relevant scaled variable is $\mu r_{s}$.

\section{A. Finite $r_{s}$, and $\mu \rightarrow 0$}

Since for small $\mu$

$$
\frac{\operatorname{erfc}(\mu x)}{x}=\frac{1}{x}-\frac{2 \mu}{\sqrt{\pi}}+\frac{2}{3} \frac{x^{2}}{\sqrt{\pi}} \mu^{3}+O\left(\mu^{5}\right),
$$

if we fix the density and let the parameter $\mu$ approach zero, we can write

$$
H=H_{\text {Coul }}+\mu H^{(1)}+\mu^{3} H^{(3)}+O\left(\mu^{5}\right),
$$

where

$$
\begin{aligned}
H^{(1)}= & \frac{N}{\sqrt{\pi}} \\
H^{(3)}= & \frac{2}{3 \sqrt{\pi}}\left(\frac{1}{2} \sum_{i \neq j}\left|\mathbf{r}_{i}-\mathbf{r}_{j}\right|^{2}-n \sum_{i} \int d \mathbf{x}\left|\mathbf{x}-\mathbf{r}_{i}\right|^{2}\right. \\
& \left.+\frac{n^{2}}{2} \int d \mathbf{x} \int d \mathbf{x}^{\prime}\left|\mathbf{x}-\mathbf{x}^{\prime}\right|^{2}\right)
\end{aligned}
$$

In Eq. (10), and in the rest of this paper, the suffix "Coul" indicates quantities of the standard uniform electron gas (jellium), with Coulomb interaction $1 / r$. Thus, for small $\mu$ we are perturbing the jellium model,

$$
\begin{aligned}
& E(\mu)=E_{\text {Coul }}+\mu E^{(1)}+\mu^{2} E^{(2)}+\ldots \\
& \Psi(\mu)=\Psi_{\text {Coul }}+\mu \Psi^{(1)}+\mu^{2} \Psi^{(2)}+\ldots
\end{aligned}
$$

Since $H^{(1)}$ is a constant, we immediately find $E^{(1)}=$ $H^{(1)}=N / \sqrt{\pi}$ and $\Psi^{(1)}=0$, which, combined with $H^{(2)}=0$, also gives $\Psi^{(2)}=0$ and $E^{(2)}=0$. Then $E^{(3)}$ is simply

$$
E^{(3)}=\left\langle\Psi_{\text {Coul }}\left|H^{(3)}\right| \Psi_{\text {Coul }}\right\rangle,
$$

and can be easily evaluated, since it is related to the plasma oscillation,,$\stackrel{24}{,}$

$$
E^{(3)}=-\frac{N}{\sqrt{\pi} \omega_{p}}=-N \frac{r_{s}^{3 / 2}}{\sqrt{3 \pi}} .
$$

Eqs. (13)-(15) hold because the expectation values of $H^{(1)}$ and $H^{(3)}$ on $\Psi_{\text {Coul }}$ exist, as it will be more explicitly shown in Eqs. (18)-(21).

Taking the energy per particle $\epsilon=E / N$, and dividing it into the non-interacting kinetic part $t_{s}=\frac{3}{10} k_{F}^{2}$ and the exchange-correlation contribution $\epsilon_{x c}$, we then have the small- $\mu$ expansion

$$
\epsilon_{x c}\left(r_{s}, \mu \rightarrow 0\right)=\epsilon_{x c}^{\mathrm{Coul}}\left(r_{s}\right)+\frac{\mu}{\sqrt{\pi}}-\frac{r_{s}^{3 / 2}}{\sqrt{3 \pi}} \mu^{3}+O\left(\mu^{4}\right) .
$$

The same result can be obtained by differentiation of $E(\mu)$ with respect to $\mu$ and by using the HelmannFeynmann theorem, which leads to the exact expression (see also Ref. 25):

$$
\frac{\partial \epsilon_{x c}}{\partial \mu}=-\frac{3}{\sqrt{\pi}} \int_{0}^{\infty} d s s^{2} e^{-\mu^{2} r_{s}^{2} s^{2}}\left[g_{x c}\left(s, r_{s}, \mu\right)-1\right],
$$


where $s=r / r_{s}$, and $g_{x c}\left(s, r_{s}, \mu\right)$ is the pair-distribution function 26.27 .28 corresponding to the Hamiltonian of Eq. (4). The evaluation of Eq. (18) at $\mu=0$, immediately gives the first-order result, $1 / \sqrt{\pi}$. Higher-order derivatives of $\epsilon_{x c}$ at $\mu=0$ can be obtained by further differentiating Eq. (18), provided that the conditions for differentiation under the integral sign are fulfilled. Since $\Psi^{(1)}=\Psi^{(2)}=0$ implies $\partial g_{x c}\left(s, r_{s}, \mu\right) /\left.\partial \mu\right|_{\mu=0}=0$ and $\partial^{2} g_{x c}\left(s, r_{s}, \mu\right) /\left.\partial \mu^{2}\right|_{\mu=0}=0$, the possibility of extracting the second and third derivatives of $\epsilon_{x c}$ at $\mu=0$ from Eq. (18) depends on whether the integrals

$$
\int_{0}^{\infty} d s s^{n}\left[g_{x c}^{\mathrm{Coul}}\left(s, r_{s}\right)-1\right]
$$

with $n=4$ and $n=6$ exist. This is the case, since $g_{x c}^{\text {Coul }}\left(s, r_{s}\right)-1$ is a well-behaved function whose oscillation-averaged part ${ }^{29}$ goes to zero as ${ }^{26.27} 1 / s^{8}$ when $s \rightarrow \infty$. We thus obtain from Eq. (18)

$$
\begin{aligned}
\left.\frac{\partial^{2} \epsilon_{x c}}{\partial \mu^{2}}\right|_{\mu=0} & =0 \\
\left.\frac{\partial^{3} \epsilon_{x c}}{\partial \mu^{3}}\right|_{\mu=0} & =\frac{6}{\sqrt{\pi}} r_{s}^{2} \int_{0}^{\infty} d s s^{4}\left[g_{x c}^{\mathrm{Coul}}\left(s, r_{s}\right)-1\right] \\
& =\frac{6}{\sqrt{\pi}} r_{s}^{2}\left(-\frac{1}{r_{s}^{2} \omega_{p}}\right)=-\frac{6}{\sqrt{3 \pi}} r_{s}^{3 / 2}
\end{aligned}
$$

in agreement with Eq. (17). We see that since $g_{x c}^{\text {Coul }}(s \rightarrow$ $\left.\infty, r_{s}\right)-1 \propto 1 / s^{8}$ no further information can be extracted from Eq. (18), or, equivalently, by going further with the expansion of Eq. (10).

One can divide $\epsilon_{x c}$ into its exchange and correlation parts, $\epsilon_{x c}=\epsilon_{x}+\epsilon_{c}$. The exchange energy $\epsilon_{x}$ has been calculated by Savin,$\frac{8}{\underline{e}}$ and is reported in Appendix $\mathrm{A}$ Its small- $\mu$ expansion is

$$
\epsilon_{x}\left(r_{s}, \mu \rightarrow 0\right)=\epsilon_{x}^{\mathrm{Coul}}\left(r_{s}\right)+\frac{\mu}{\sqrt{\pi}}-\frac{3 \alpha r_{s}}{2 \pi} \mu^{2}+O\left(\mu^{4}\right),
$$

where $\alpha=(4 / 9 \pi)^{1 / 3}$. The $\mu \rightarrow 0$ behavior of $\epsilon_{c}=$ $\epsilon_{x c}-\epsilon_{x}$, is then

$$
\epsilon_{c}\left(r_{s}, \mu \rightarrow 0\right)=\epsilon_{c}^{\mathrm{Coul}}\left(r_{s}\right)+\frac{3 \alpha r_{s}}{2 \pi} \mu^{2}-\frac{r_{s}^{3 / 2}}{\sqrt{3 \pi}} \mu^{3}+O\left(\mu^{4}\right) .
$$

Notice that if we divide the pair-distribution function $g_{x c}$ into its exchange and correlation parts, $g_{x c}\left(s, r_{s}, \mu\right)=$ $g_{x}(s)+g_{c}\left(s, r_{s}, \mu\right)$, we have

$$
\begin{aligned}
& \frac{\partial \epsilon_{x}}{\partial \mu}=-\frac{3}{\sqrt{\pi}} \int_{0}^{\infty} d s s^{2} e^{-\mu^{2} r_{s}^{2} s^{2}}\left[g_{x}(s)-1\right], \\
& \frac{\partial \epsilon_{c}}{\partial \mu}=-\frac{3}{\sqrt{\pi}} \int_{0}^{\infty} d s s^{2} e^{-\mu^{2} r_{s}^{2} s^{2}} g_{c}\left(s, r_{s}, \mu\right) .
\end{aligned}
$$

(This follows directly from the Hellmann-Feynmann theorem and from the fact that $g_{x}$ corresponds to the noninteracting gas and thus does not depend on $\mu$.) If we take the limit $\mu \rightarrow 0$ of Eqs. (24) and (25) we recover the first-order result in Eqs. (22) and (23). However, higher-order derivatives at $\mu=0$ of $\epsilon_{x}$ and $\epsilon_{c}$ cannot be obtained by differentiating Eqs. (24) and (25). This is due to the long-range tail of $g_{x}(s)-1$ and $g_{c}^{\text {Coul }}\left(s, r_{s}\right)$ : when $s \rightarrow \infty$ they both approach zero as $\frac{26.27 .28}{1 / s^{4}}$. Thus, integrals of the kind $\int_{0}^{\infty} s^{4}\left[g_{x}-1\right]$ and $\int_{0}^{\infty} s^{4} g_{c}^{\text {Coul }}$ diverge. The long-range tails of $g_{x}(s)-1$ and $g_{c}^{\operatorname{Coul}}\left(s, r_{s}\right)$ exactly cancel 26.27 .28 in $g_{x c}^{\mathrm{Coul}}\left(s, r_{s}\right)-1$. This is why, at small $\mu$, both $\epsilon_{x}$ and $\epsilon_{c}$ have terms $\propto \mu^{2}$ which cancel out in $\epsilon_{x c}$.

\section{B. Finite $\mu$, and $r_{s} \rightarrow 0$}

If we use the relevant scaled units $\mathbf{s}=\mathbf{r} / r_{s}$ and we let $r_{s}$ approach zero, the potential has the expansion

$$
\left.\frac{1}{r_{s}} \frac{\operatorname{erfc}\left(\mu r_{s} s\right)}{s}\right|_{r_{s} \rightarrow 0}=\frac{1}{r_{s}}\left(\frac{1}{s}-\frac{2 \mu}{\sqrt{\pi}} r_{s}+\frac{2}{3} \frac{\mu^{3} s^{2}}{\sqrt{\pi}} r_{s}^{3}+\ldots\right),
$$

which has the Coulomb interaction as leading term. We are thus approaching again the jellium model, so that Eq. (23) is also valid for finite $\mu$ and $r_{s} \rightarrow 0$.

In Eq. (23) the relevant scaled variable is $\mu \sqrt{r_{s}}$. This can be understood in the following way. The Coulomb gas presents screening effects at lenghts $r \gtrsim 1 / q_{\mathrm{TF}} \propto$ $\sqrt{r_{s}}$, where $q_{\mathrm{TF}}$ is the Thomas-Fermi wave vector. Since the erfc function amounts to some sort of artificial screening at lenghts $r \gtrsim \mu^{-1}$, the Thomas-Fermi screening appears, exactly as in the Coulomb gas, when $\sqrt{r_{s}} \ll \mu^{-1}$

\section{C. $\mu r_{s} \gg 1$}

When $\mu \rightarrow \infty$, the potential terms of Eqs. (5)-(17) rapidly vanish $\left(V^{\mathrm{SR}} \sim e^{-\mu^{2} r^{2}}\right)$. In this regime we can treat the whole potential as a perturbation to the noninteracting Fermi gas.

The first-order (in the potential) correction to the noninteracting energy $t_{s}$ gives $\epsilon_{x}$ of Appendix The secondorder term can be computed by standard RayleighSchrödinger perturbation theory

$$
\epsilon^{(2)}=-\frac{1}{N} \sum_{n \neq 0} \frac{\left\langle 0\left|V^{\mathrm{SR}}\right| n\right\rangle\left\langle n\left|V^{\mathrm{SR}}\right| 0\right\rangle}{E_{n}-E_{0}} .
$$

As in the case of jellium, $\epsilon^{(2)}$ is the sum of a direct term and of a second-order exchange term, 24 which in Fourier space read 


$$
\begin{aligned}
& \epsilon_{\mathrm{dir}}^{(2)}=-\frac{3}{16 \pi^{5}} \int d \mathbf{q}\left(\frac{1-e^{-q^{2} k_{F}^{2} / 4 \mu^{2}}}{q^{2}}\right)^{2} \int_{|\mathbf{k}+\mathbf{q}|>1} d \mathbf{k} \int_{|\mathbf{p}+\mathbf{q}|>1} d \mathbf{p} \frac{\theta(1-k) \theta(1-p)}{q^{2}+\mathbf{q} \cdot(\mathbf{k}+\mathbf{p})} \\
& \epsilon_{\mathrm{ex}}^{(2)}=\frac{3}{32 \pi^{5}} \int d \mathbf{q} \frac{1-e^{-q^{2} k_{F}^{2} / 4 \mu^{2}}}{q^{2}} \int_{|\mathbf{k}+\mathbf{q}|>1} d \mathbf{k} \int_{|\mathbf{p}+\mathbf{q}|>1} d \mathbf{p} \frac{1-e^{-|\mathbf{q}+\mathbf{k}+\mathbf{p}|^{2} k_{F}^{2} / 4 \mu^{2}}}{|\mathbf{q}+\mathbf{k}+\mathbf{p}|^{2}} \cdot \frac{\theta(1-k) \theta(1-p)}{q^{2}+\mathbf{q} \cdot(\mathbf{k}+\mathbf{p})} .
\end{aligned}
$$

Here all the momenta are expressed in units of $k_{F}=$ $\left(\alpha r_{s}\right)^{-1}$, and $\theta(x)$ is the Heaviside step function. Now, consider the case $\mu r_{s} \rightarrow \infty$ and divide the integral over $q$ in Eqs. (27) and (28) into two parts:

$$
\int_{0}^{\infty} d q=\int_{0}^{q_{1}} d q+\int_{q_{1}}^{\infty} d q, \quad \text { with } 1 \ll q_{1} \ll \mu r_{s} .
$$

In the first part, when $q \in\left[0, q_{1}\right]$, we can write

$$
\begin{aligned}
1-e^{-q^{2} k_{F}^{2} / 4 \mu^{2}} & \approx \frac{q^{2} k_{F}^{2}}{4 \mu^{2}}, \\
1-e^{-|\mathbf{q}+\mathbf{k}+\mathbf{p}|^{2} k_{F}^{2} / 4 \mu^{2}} & \approx \frac{|\mathbf{q}+\mathbf{k}+\mathbf{p}|^{2} k_{F}^{2}}{4 \mu^{2}}
\end{aligned}
$$

(since $q_{1} \ll \mu r_{s}$, and the integrals of Eqs. (27) and (28) are restricted to the domain $|\mathbf{k}| \leq 1,|\mathbf{p}| \leq 1)$. Equations (27) and (28) then reduce to integrals of the same kind, which can be summed to yield

$$
\begin{aligned}
\epsilon_{|\mathbf{q}| \leq q_{1}}^{(2)}= & \frac{-3}{32 \pi^{5}}\left(\frac{k_{F}^{2}}{4 \mu^{2}}\right)^{2} \int_{|\mathbf{q}| \leq q_{1}} d \mathbf{q} \int_{|\mathbf{k}+\mathbf{q}|>1} d \mathbf{k} \\
& \times \int_{|\mathbf{p}+\mathbf{q}|>1} d \mathbf{p} \frac{\theta(1-k) \theta(1-p)}{q^{2}+\mathbf{q} \cdot(\mathbf{k}+\mathbf{p})},
\end{aligned}
$$

i.e., they give a term which vanishes as $\left(\mu r_{s}\right)^{-4}$. In the second part, $q \in\left[q_{1}, \infty\right)$, having chosen $q_{1} \gg 1$, we can write

$$
\begin{aligned}
& |\mathbf{q}+\mathbf{k}+\mathbf{p}|^{2} \approx q^{2}, \\
& \int_{|\mathbf{k}+\mathbf{q}|>1} d \mathbf{k} \int_{|\mathbf{p}+\mathbf{q}|>1} d \mathbf{p} \frac{\theta(1-k) \theta(1-p)}{q^{2}+\mathbf{q} \cdot(\mathbf{k}+\mathbf{p})} \approx\left(\frac{4 \pi}{3}\right)^{2} \frac{1}{q^{2}} .
\end{aligned}
$$

Equations (27) and (28) again reduce to integrals of the same kind, which can be summed to yield

$$
\epsilon_{|\mathbf{q}| \geq q_{1}}^{(2)}=-\frac{2}{3 \pi^{2}} \int_{q_{1}}^{\infty} d q\left(\frac{1-e^{-q^{2} k_{F}^{2} / 4 \mu^{2}}}{q^{2}}\right)^{2} .
$$

The right-hand side of Eq. (29) can be evaluated analytically and then expanded for $\mu r_{s} \rightarrow \infty$. Its leading term is (correctly) independent of $q_{1}$ and equals $-\frac{\sqrt{2}-1}{4 \sqrt{\pi}}\left(\mu r_{s}\right)^{-3}$. We thus have

$$
\left.\epsilon_{c}\left(r_{s}, \mu\right)\right|_{\mu r_{s} \gg 1}=-\frac{A}{\left(\mu r_{s}\right)^{3}}+\frac{B}{\left(\mu r_{s}\right)^{4}}+\ldots
$$

with $A \approx \frac{\sqrt{2}-1}{4 \sqrt{\pi}} \approx 0.0584$ Hartree.

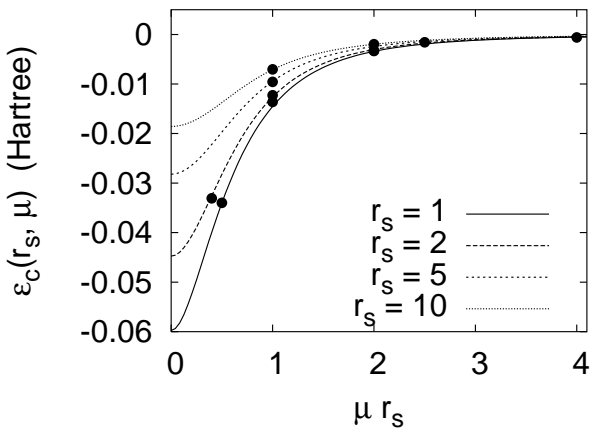

FIG. 3: Correlation energy of the short-range interacting gas as a function of $\mu r_{s}$, for different densities. Our fitting function (lines) is compared with our DMC data (dots). The error bars are comparable with the symbol sizes.

Since the perturbation series expansion whose secondorder term corresponds to Eq. [26) is done with respect to the whole potential $V^{\mathrm{SR}}$ and not with respect to the parameter $\mu$, higher-order terms could also contribute to the value of $A$. For this reason, in our parametrization of $\epsilon_{c}\left(r_{s}, \zeta\right) A$ is left as a free parameter, to be optimized with a fit on the DMC data. We expect to find a value of $A$ of the same order of the one estimated with Eq. (26), since the potential $V^{S R}$ vanishes very rapidly as $\mu \rightarrow \infty$, so that the higher-order-term contribution to $A$ should be small.

\section{ANALYTIC REPRESENTATION OF THE CORRELATION ENERGY}

An accurate and simple analytic representation of the correlation energy $\epsilon_{c}\left(r_{s}, \mu\right)$ can be obtained by a Padé form which interpolates between the limiting behaviors given by our Eqs. (23) and (30), and contains some free parameters to fit our DMC data. We write

$\epsilon_{c}\left(r_{s}, \mu\right)=\frac{\epsilon_{c}^{\mathrm{Coul}}\left(r_{s}\right)\left[1+b_{1}\left(r_{s}\right) \mu\right]}{1+b_{1}\left(r_{s}\right) \mu+b_{2}\left(r_{s}\right) \mu^{2}+b_{3}\left(r_{s}\right) \mu^{3}+b_{4}\left(r_{s}\right) \mu^{4}}$, 
where

$$
\begin{aligned}
& b_{1}=\left(b_{3}-\frac{r_{s}^{3 / 2}}{\sqrt{3 \pi}} \frac{1}{\epsilon_{c}^{\text {Coul }}}\right) \frac{1}{b_{2}} \\
& b_{2}=-\frac{3}{2 \pi} \frac{\alpha r_{s}}{\epsilon_{c}^{\text {Coul }}} \\
& b_{4}=-b_{1} \epsilon_{c}^{\text {Coul }} \frac{r_{s}^{3}}{A},
\end{aligned}
$$

and $\epsilon_{c}^{\operatorname{Coul}}\left(r_{s}\right)$ is one of the standard parametrizations $\frac{18,19,20}{10}$ of the correlation energy of the unpolarized jellium. Here we used the parametrization of Perdew and Wang ${ }^{20}$ The two parameters $b_{3}\left(r_{s}\right)$ and $A$ are fixed by a two-dimensional $\left(r_{s}, \mu\right)$ best fit to our DMC data. We find:

$$
\begin{aligned}
& b_{3}\left(r_{s}\right)=1.27 r_{s}^{7 / 2} \\
& A=0.03579 .
\end{aligned}
$$

This fit yields a reduced $\chi^{2}$ of 2.7. In Fig. 3] we show our DMC data together with the fitting function for different values of $r_{s}$. Notice that our analytic $\epsilon_{c}\left(r_{s}, \mu\right)$ does not break down at high $\left(r_{s} \rightarrow 0\right)$ or low $\left(r_{s} \rightarrow \infty\right)$ densities, being constrained by exact behaviors.

\section{CONCLUSIONS AND PERSPECTIVES}

We have presented a comprehensive numerical and analytic study of the ground-state energy of a (spin unpolarized) uniform electron gas with modified, short-rangeonly electron-electron interaction $\operatorname{erfc}(\mu r) / r$, as a function of the cutoff parameter $\mu$ and of the electronic density. Our chief goal has been the publication, in a convenient form for application, of a reliable local density functional for the correlation energy of this model system, which (i) fits the results of our quantum Monte Carlo simulations and (ii) automatically incorporates exact limits. Such a functional is a crucial ingredient for some recently proposed "mixed schemes", which exploit the DFT only for the short-range part of the electron-electron interaction. In this context the natural extension of this study will be the generalization of our functional to the spinpolarized case.

What we obtained in this paper is not the only possible short-range local-density functional of interest to "mixed schemes". In some of them ${ }^{9}$ the DFT treatment of the short-range part is handled through another functional $\bar{E}_{x c}^{\mathrm{SR}}[n]$, defined as the difference between the standard exchange-correlation energy functional (corresponding to the Coulomb interaction) and a long-range-only functional

$$
\bar{E}_{x c}^{\mathrm{SR}}[n]=E_{x c}[n]-E_{x c}^{\mathrm{LR}}[n] .
$$

Another direction of future work will thus be the study of the uniform electron gas with a long-range-only interaction of the form of Eq. (2), and, possibly, other modified interactions proposed in the same spirit 30

\section{Acknowledgments}

We thank S. Baroni, A. Savin, and J. Toulouse for useful discussions, and gratefully acknowledge financial support from the Italian Ministry of Education, University and Research (MIUR) through COFIN 2003-2004 and the allocation of computer resources from INFM Iniziativa Calcolo Parallelo.

\section{APPENDIX A: EXCHANGE ENERGY}

The exchange energy corresponding to the Hamiltonian (4) has been calculated by Savin in Ref. 8, and is equal to

$$
\begin{aligned}
\epsilon_{x}\left(r_{s}, \mu\right)= & -\frac{2}{\pi} k_{F}\left\{\frac{3}{8}-a\left[\sqrt{\pi} \operatorname{erf}\left(\frac{1}{2 a}\right)-3 a+4 a^{3}\right.\right. \\
& \left.\left.+\left(2 a-4 a^{3}\right) e^{-1 / 4 a^{2}}\right]\right\},
\end{aligned}
$$

with $a=\mu /\left(2 k_{F}\right)$. The exchange energy thus satisfies

$$
\epsilon_{x}\left(r_{s}, \mu\right)=r_{s}^{-1} f\left(\mu r_{s}\right) .
$$

${ }^{1}$ W. Kohn, Rev. Mod. Phys. 71, 1253 (1999).

2 A.E. Mattsson, Science 298, 759 (2002).

${ }^{3}$ C. Fiolhais, F. Nogueira, and M. Marques (eds.), A Primer in Density Functional Theory (Springer-Verlag, Berlin, 2003).

4 H. Rydberg, M. Dion, N. Jacobson, E. Schröder, P. Hyldgaard, S. I. Simak, D.C. Langreth, and B.I. Lundqvist, Phys. Rev. Lett. 91, 126402 (2003).

5 J. Dobson and J. Wang, Phys. Rev. B 69, 235104 (2004).

6 J. Tao, J.P. Perdew, V.N. Staroverov, and G.E. Scuseria, Phys. Rev. Lett. 91, 146401 (2003); J.P. Perdew, J. Tao, V.N. Staroverov, and G.E. Scuseria, J. Chem. Phys. 120, 6898 (2004).
7 J. Ireta, J. Neugebauer, and M. Scheffler, On the accuracy of DFT for describing hydrogen bonds: dependence on the bond directionality, preprint.

8 A. Savin, in Recent Developments and Applications of Modern Density Functional Theory, edited by J.M. Seminario (Elsevier, Amsterdam, 1996).

9 R. Pollet, A. Savin, T. Leininger, and H. Stoll, J. Chem. Phys. 116, 1250 (2002); T. Leininger, H. Stoll, H.-J. Werner, and A. Savin, Chem. Phys. Lett. 275, 151 (1997).

10 W. Kohn, Y. Meir, D.E. Makarov, Phys. Rev. Lett. 80, 4153 (1998).

11 J. Heyd, G.E. Scuseria, and M. Ernzerhof, J. Chem. Phys. 118, 8207 (2003); J. Heyd and G.E. Scuseria, J. Chem. 
Phys. 120, 7274 (2004).

12 J.P. Perdew, A. Savin, and K. Burke, Phys. Rev. A 51, 4531 (1995); K. Burke, J.P. Perdew, and M. Ernzerhof, J. Chem. Phys. 109, 3760 (1998).

13 M. Fuchs and X. Gonze, Phys. Rev. B 65, 235109 (2002).

14 see also the comment of M. Fuchs, K. Burke, Y.-M. Niquet, and X. Gonze, Phys. Rev. Lett. 90, 189701 (2003).

15 A. Savin, F. Colonna, and R. Pollet, Int. J. Quantum Chem. 93, 166 (2003). See also Refs 8910.

16 J. Toulouse, F. Colonna, and A. Savin, in preparation.

17 D.M. Ceperley and B.J. Alder, Phys. Rev. Lett. 45, 566 (1980).

18 S.H. Vosko, L. Wilk, and M. Nusair, Can. J. Phys. 58, 1200 (1980).

19 J.P. Perdew and A. Zunger, Phys. Rev. B 23, 5048 (1981).

20 J.P. Perdew and Y. Wang, Phys. Rev. B 45, 13244 (1992).

21 For a recent review on fixed-node diffusion Monte Carlo and further references, see M. Foulkes, L. Mitas, R. Needs and G. Rajagopal, Rev. Mod. Phys. 73, 33-83 (2001).

${ }^{22}$ Y. Kwon, D. M. Ceperley and R. M. Martin, Phys. Rev.
B 586800 (1998).

23 M.P. Allen and D.J. Tildesley, Computer simulation of liquids (Clarendon Press, Oxford, 1987).

24 see, e.g., D. Pines and P. Nozières, Theory of Quantum Liquids (Benjamin, New York, 1966).

25 R. Pollet, F. Colonna, T. Leininger, H. Stoll, H.-J. Werner, and A. Savin, Int. J. Quantum Chem. 91, 84 (2003).

26 P. Gori-Giorgi, F. Sacchetti, and G.B. Bachelet, Phys. Rev. B 61, 7353 (2000); 66, 159901(E) (2002).

27 P. Gori-Giorgi and J.P. Perdew, Phys. Rev. B 66, 165118 (2002).

28 J.P. Perdew and Y. Wang, Phys. Rev. B 46, 12947 (1992); 56, 7018(E) (1997).

29 Energetically unimportant oscillating terms are dealt with the evaluation of integrals of the kind: $\lim _{a \rightarrow 0} \int_{0}^{\infty} e^{-a x} \cos (x) d x$.

30 A. Savin and H.-J. Flad, Int. J. Quantum Chem. 56, 327 (1995); J. Toulouse, A. Savin, and H.-J. Flad, Int. J. Quantum Chem., to appear. 